Mr. G. P. Crowden referred to the work of the British Medical Association and said that further efforts are needed to strengthen the link between laboratory and kitchen. He suggested the establishment of a central bureau to give information on matters relating to family nutrition, and that pur. veyors of foodstuffs might assist purchasing and distribution by offering attractive terms to housewives for weekly supplies of items in the dietary scales recommended.
Dr. G. C. M'Gonigle thought the E.S.G. report a notable contribution to family economics. He has analysed many budgets of the lowly paid and unemployed, and has found that the relatively low standard of the B.M.A. minimum dietary cannot be achieved under a family income of 55-65s. a week. High rent is a great obstacle. Contrary to popular belief, he found that the average working-class woman has a sound empirical knowledge of practical dietetics, and to the limit of her purse buys wisely and well.

\title{
Micro-Ray Communication
}

$\mathrm{I}^{\mathrm{N}}$ engineering, the progress of the evolution of inventions seldom proceeds along a straight line, each step leading directly and logically to its successor. The evolution often proceeds in a looped path, new inventions apparently returning to an earlier stage of development before making a new start in another direction. This is illustrated in a paper read by W. L. McPherson and E. H. Ullrich to the Institution of Electrical Engineers on January 30.

In describing the recent commercialisation of micro-ray transmissions for radio communication, the authors point out that it was with micro-rays that Hertz in 1887 performed the classic experiments which are generally admitted to have led to the wireless communication of to-day. Hertz succeeded in generating by means of a spark transmitter wavelengths of $30 \mathrm{~cm}$., and in proving conclusively that the radiation due to such circuits followed the optical laws of reflection, refraction and propagation. He thus verified Clerk Maxwell's work done twenty-two years previously. Hertz's investigations read almost like an experimental and theoretical study of light. They include the measurements of the angles of incidence and reflection from plane sheets of metal, reflection from curved metallic mirrors of definite focal length, refraction through prisms of pitch and studies in transparency and opacity. Owing to lack of sensitivity on the receiving side, Hertz's experiments bore no immediate fruit although scientific workers were greatly interested in them.

The invention of the large aerial and of tuning, and the success of medium and long wave operation, led to practically total neglect of micro-ray technique until 1919, when Barkhausen and Kurz discovered a new type of oscillatory circuit which generated wave. lengths down to about $43 \mathrm{~cm}$. The first large-scale demonstration of modern micro-ray working was made between St. Margaret's Bay, near Dover, and Escalles, near Calais, a distance of $22 \cdot 1$ miles. The wave-length used was only $18 \mathrm{~cm}$. and so the waves radiated like light waves, permitting the use of reflecting mirrors. In January 1934 a micro-ray link for commercial service was opened between the aerodromes of Lympne in England and St. Inglevert in France, covering a distance of thirty-five miles. It provides a duplex service on radio-telephony, teleprinter or Morse telegraphy as required. The wavelength is $17.4 \mathrm{~cm}$., which is the shortest of any station in the world.

Experiments have shown that atmospherics of the ordinary type are never heard on the St. Margaret's circuit, although a few sharp clicks are sometimes noticed the origin of which has not yet been traced.
Thunderstorms occurring even in sight of the receiving station cause no interference. The ignition systems of aircraft or motor-boats also have no effect. It was found that the strength of the received signals did not remain constant. On three occasions the signal was uncommercial in telegraphy for periods of about two minutes. When a $600 \mathrm{~cm}$. wave-length was used, no variation of the received signal was ever detected.

Diagrams are given of the working signal current operating the teleprinter at Lympne and of the state of the tides at the same times. Although the effect of the tides may be masked in many instances by other fading causes, the records suggest that they are a factor in causing fading. In nine cases out of ten, the turn of the tide, during a week's record, was accompanied by a marked change in the slope of the signal current curve. This supports the hypothesis that there exists interference due to the indirect ray reflected at the sea surface.

The authors conclude that the primary condition for good micro-ray working is a thoroughly well-mixed and homogeneous atmosphere. In the summer, currents of hot air probably create 'pockets' of very different refractive power from the rest of the atmo. sphere, so that the direction of transmission may be violently changed and unusual attenuation intro. duced. In summer, the passage of a cloud across the sun's rays gives a temperature 'kick' the magnitude of which is different when above land than when above the sea, owing to the difference in reradiation in the two cases. The path of the St. Margaret'sEscalles link is at first sight simpler, as it is nearly all above the sea; but at the faces of the cliffs near the stations strong currents of hot air often arise, and this would introduce a compound lens effect in the path of the transmission rays.

The results obtained by the practical working of the two 'links' described in the paper may be sum. marised as follows. The most stable micro-ray conditions coincide with very stable atmospheric conditions, as judged by thermometer and barometer. A high wind is almost invariably accompanied with good micro-ray transmission. The settling of a heavy bank of fog has been accompanied by very severe and rapid changes in the reception, followed by stability when the fog bank has ceased to move. Radio waves, six metres in length, are much more stable than micro-waves over optical paths across the Straits of Dover. It is much more difficult to tap micro-wave communications than those which use longer wave-lengths. Hence when secrecy is necessary, as in military operations, they are more desirable. 